\title{
FORMULATION OF SUSPENDING VEHICLES FOR EXTEMPORANEOUS ORAL LIQUID \\ COMPOUNDING WITH DILTIAZEM HYDROCHLORIDE AS A DRUG MODEL
}

\author{
SUTRIYO*, ZAHRA MEILIA NISA
}

Department of Pharmacy, Universitas Indonesia, Depok, 16424, Indonesia. Email: sutriyo@farmasi.ui.ac.id

Received: 04 May 2018, Revised: 29 September 2018, Accepted: 15 November 2018

\section{ABSTRACT}

Objective: This study aimed to determine the optimum concentrations of a suspending vehicle and develop a physically and chemically stable formulation containing diltiazem hydrochloride (DTZ).

Methods: Stability testing was performed for 30 days in selected suspending vehicle formulas (Formulas A and E). Physical stability tests were performed at room temperature, and physical properties (odor and color) and pH values of suspensions were evaluated.

Results: The oral suspensions of DTZ were white and had a drug-like odor, and the pH had significantly decreased during storage. Chemical stability tests of suspensions were performed at room temperature and $4^{\circ} \mathrm{C} \pm 2^{\circ} \mathrm{C}$ using ultraviolet-visible spectrophotometry.

Conclusion: The concentrations of DTZ in oral suspensions fluctuated during the storage period, suggesting that the oral suspensions of DTZ are physically stable but not chemically stable during storage.

Keywords: Diltiazem hydrochloride, Suspending vehicle, Extemporaneous oral liquid formulation, Stability.

(C) 2018 The Authors. Published by Innovare Academic Sciences Pvt Ltd. This is an open access article under the CC BY license (http://creativecommons. org/licenses/by/4. 0/) DOI: http://dx.doi.org/10.22159/ijap.2018.v10s1.51

\section{INTRODUCTION}

Extemporaneous compounding in liquid vehicles facilitates the regulation of drug dosing in patients with swallowing difficulties and can provide greater ease for patients taking the drug [1]. Extemporaneous oral liquid compounding also has several advantages including masking of the bitter taste and uniformity of drug doses. In particular, extemporaneous oral liquid compounding will be of great use in pediatric patients $[2,3]$.

Liquid oral formulation is comparatively quick to prepare and allow in the dosage form [4]. The lack of oral liquid drug formulations is problematic for patients with swallowing difficulties such as pediatric and geriatric patients. These patients require the production of extemporaneous compounding in liquid vehicles that are compatible with oral administration [5]. Accordingly, previous pharmaceutical studies show that formulations of extemporaneous compounding in liquid vehicles can assist patients with swallowing difficulty [6].

According to surveys conducted at several hospitals in Australia by Stewart and Tucker, 116 types of prescribed drugs were made into 270 forms of extemporaneous compounding in liquid vehicles, and these are particularly used for pediatric patients. The preparation of extemporaneous compounding in liquid vehicles is relatively easy and can be achieved by simply dispersing the active drug agent into a suspending vehicle [2].

In Australia, Europe, and the United States, suspending vehicles for extemporaneous oral liquid compounding have been circulated in the market as Ora-Plus, which is a physically stable suspending vehicle with an elegant appearance and a uniform dose [6].

Herein, since the Ora-Plus vehicle is not available in Indonesia, we developed a suspending vehicle formulation for extemporaneous compounding using diltiazem hydrochloride (DTZ) as a drug model. This compound is a calcium channel blocker that is used for the treatment of angina pectoris and hypertension, particularly in pediatric and geriatric patients. It is highly protein bound $70-90 \%$ and undergoes extensive first-pass metabolism with relatively poor oral bioavailability of $40 \%$ [7]. We selected this drug as an active agent because it is compatible with suspending vehicles, which are intended for the treatment of pediatric and geriatric patients [8]. The present suspending vehicles were then evaluated for appearance, physical stability, and dose uniformity in combination with the active ingredient.

\section{METHODS}

Formulation of suspending vehicles

To produce suspending vehicles, suspending agents were dispersed in water and then stirred using a homogenizer. Preservative agents were then dissolved in solutions containing sufficient amounts of propylene glycol and were then added to suspending solutions. Mixtures were then homogenized using a homogenizer. Subsequently, buffer was dissolved in aquadest and was added to mixtures of suspending and preservative agents. After mixing using a homogenizer, dimethicone was added and mixed with the suspending agent, buffer, and preservative. Calcium sulfate was then dissolved in residual propylene glycol and was incorporated into the mixture using the homogenizer. Finally, sufficient volumes of aquadest were added quickly with stirring (Table 1).

\section{Evaluations of suspending vehicles}

Sedimentation volumes

Sedimentation volumes are ratios of the final volume of the sediment formed to the initial volume of the suspension vehicle before settling and are calculated using the following formula:

$$
\mathrm{F}(\%)=100 \mathrm{Vu} / \mathrm{Vo} \text {, }
$$

Where, $\mathrm{F}$ is the sedimentation volume, Vu is the final volume of sediment, and Vo is the initial volume of the suspension vehicle before settling.

To determine sedimentation volumes, 50 -mL aliquots of the suspending vehicle were transferred to a measuring cup and sediment formation was monitored during storage for $1,5,10,15,20,25$, and 30 days [9]. 
Table 1: Suspending vehicle formulations

\begin{tabular}{|c|c|c|c|c|c|c|c|}
\hline \multirow[t]{2}{*}{ Material components } & \multicolumn{7}{|c|}{ Formula (\%) } \\
\hline & A & B & C & D & $\mathbf{E}$ & $\mathbf{F}$ & G \\
\hline Microcrystalline cellulose & 0.5 & 3 & 3 & 3 & - & - & - \\
\hline Xanthan gum & 0.1 & - & 0.1 & - & 0.1 & - & 0.1 \\
\hline Carrageenan & 0.5 & - & - & 0.75 & - & 0.75 & 0.75 \\
\hline Calcium sulfate & 0.4 & 0.4 & 0.4 & 0.4 & 0.4 & 0.4 & 0.4 \\
\hline Methylparaben & 0.03 & 0.03 & 0.03 & 0.03 & 0.03 & 0.03 & 0.03 \\
\hline Potassium sorbate & 0.1 & 0.1 & 0.1 & 0.1 & 0.1 & 0.1 & 0.1 \\
\hline Citric acid & 0.3 & 0.3 & 0.3 & 0.3 & 0.3 & 0.3 & 0.3 \\
\hline Sodium dihydrogen phosphate & 0.1 & 0.1 & 0.1 & 0.1 & 0.1 & 0.1 & 0.1 \\
\hline Dimethicone & 0.5 & 0.5 & 0.5 & 0.5 & 0.5 & 0.5 & 0.5 \\
\hline Propylene glycol & 10 & 10 & 10 & 10 & 10 & 10 & 10 \\
\hline Aquadest & ad 100 & ad 100 & ad 100 & ad 100 & ad 100 & ad 100 & ad 100 \\
\hline
\end{tabular}

\section{Redispersion}

$50 \mathrm{~mL}$ aliquots of suspending vehicle were placed into glass bottles and were stored at room temperature for 1 month. On days 1, 5, 10, $15,20,25$, and 30 , redispersion tests were performed by shaking and observing redispersion of the sediments that formed during storage [9]. Comparisons were made in terms of numbers of shakes required to disperse sediments.

\section{Viscosity tests}

Viscosity was tested using a Brookfield viscometer at $100 \mathrm{rpm}$ with spindles 1 or 2 depending on the viscosity of the suspending vehicle formulas [10]. Experiments were performed in triplicate and viscosities were expressed as means [9].

\section{Particle size tests}

Particle size testing was performed using a Mastersizer tool, which calculates particle size distributions in solutions of micron-sized particles. Experiments were performed 3 times and the data are expressed as mean particle size distributions for each suspending vehicle.

\section{pH tests}

Suspending vehicles were placed in glass beakers and $\mathrm{pH}$ values were measured using a $\mathrm{pH}$ meter that had been calibrated using buffer solutions at $\mathrm{pH} 4$ and 7 [11].

\section{Selection of suspending vehicle formulas}

Active substance in the form of diluted DTZ tablets was incorporated into suspending vehicle formulas, and selected suspending vehicle formulas A and E were produced. Physical and chemical stability tests were conducted after 1 month, and DTZ levels in suspending vehicles were determined using ultraviolet (UV)-visible spectrophotometry.

To produce oral suspensions of DTZ, 24 tablets containing $30 \mathrm{mg}$ of dilute DTZ were added to $60-\mathrm{mL}$ aliquots of suspending vehicle to give a concentration of $12 \mathrm{mg} / \mathrm{mL}$ (Table 2). Mixtures were then shaken until DTZ powder was evenly dispersed in suspending vehicle. Sedimentation volume tests, redispersion tests, viscosity tests, $\mathrm{pH}$ tests, and physical and chemical stability tests were then performed at the indicated times.

\section{Physical stability tests}

Physical stability testing was performed at room temperature $\left(25^{\circ} \mathrm{C} \pm 2^{\circ} \mathrm{C}\right)$ with samples of selected suspending vehicle mixtures containing DTZ. Organoleptic observations (odor and color) were observed and $\mathrm{pH}$ values of vehicles were tested after $0,3,5,10,15,20$, 25 , and 30 days [1].

\section{Determination of DTZ concentrations}

\section{Preparation of DTZ standard solution}

Standard DTZ powder was weighed and 10-mg samples were added to $100-\mathrm{mL}$ measuring flasks. Subsequently, 50-mL aliquots of aquadest
Table 2: DTZ contents in suspending vehicle

\begin{tabular}{lll}
\hline Material components & Total & Final concentration \\
\hline DTZ tablet $30 \mathrm{mg}$ & 24 tablet & $12 \mathrm{mg} / \mathrm{mL}$ \\
Selected suspending vehicle & Add $60 \mathrm{~mL}$ & \\
\hline
\end{tabular}

DTZ: Diltiazem hydrochloride

were added to the measuring flasks and were sonicated for $15 \mathrm{~min}$. Flasks were then filled to volume with aquadest to achieve a standard concentration of $100-\mu \mathrm{g} / \mathrm{mL}$ DTZ, and $10-\mathrm{mL}$ aliquots were then placed into $100 \mathrm{~mL}$ measuring flasks and were diluted 10 -fold with aquadest to a final concentration of $10 \mu \mathrm{g} / \mathrm{mL}$ [12].

\section{Determination of wavelength maxima}

Wavelength maxima of the standard $10-\mu \mathrm{g} / \mathrm{mL}$ DTZ solution were determined using a UV-visible spectrophotometer at a wavelength range of 200-400 $\mathrm{nm}$. Aquadest was used as the blank solution and a maximum wavelength of $236 \mathrm{~nm}$ was identified for the standard DTZ solution [12].

\section{DTZ calibration curve}

Standard solutions containing $100-\mu \mathrm{g} / \mathrm{mL}$ DTZ were diluted into 6-, 8-, 10-, 12-, 14-, and 16- $\mu \mathrm{g} / \mathrm{mL}$ solutions. Absorbance was then determined using a UV-visible spectrophotometer at $236 \mathrm{~nm}$. The relationship between absorbance and concentration was expressed as a linear regression equation and is presented as a calibration curve [12].

\section{Determination of DTZ tablet concentrations}

A total of 20 tablets containing $30 \mathrm{mg}$ of DTZ each were weighed and then powdered, and average weights were then calculated. An equivalent powder containing $10 \mathrm{mg}$ of DTZ was dissolved in $30 \mathrm{~mL}$ of aquadest using sonication and was then diluted to volume in $100 \mathrm{~mL}$ of aquadest. Subsequently, 5 -mL aliquots of the first filtrates were removed and diluted to $10 \mu \mathrm{g} / \mathrm{mL}$, and DTZ concentrations were determined in triplicate at $236 \mathrm{~nm}$ using a UV-visible spectrophotometer [12].

\section{Determination of DTZ concentrations in suspensions}

Suspensions of $5 \mathrm{~mL}$ containing $60 \mathrm{mg}$ of DTZ were extracted in a separating funnel using $50 \mathrm{~mL}$ of chloroform. Mixtures were shaken for about $1 \mathrm{~min}$ and after separation from suspension phases, organic phases were taken and filtered through Whatman filter paper No. 42. Filtrates were then dried in a vaporizer plate in an acid cupboard until the chloroform had evaporated entirely. The resulting residues were then added to 50-mL measuring flasks and were diluted to volume in aquadest. Further, dilutions were performed with 1-mL aliquots of solution in 100-mL measuring flasks to achieve a concentration of $1 \mu \mathrm{g} / \mathrm{mL}$. DTZ concentrations were then determined at a wavelength of $238.4 \mathrm{~nm}$ using a UV-visible spectrophotometer in triplicate [13]. 
Chemical stability test

Selected suspending vehicles containing DTZ tablets were stored at $4^{\circ} \mathrm{C} \pm 2^{\circ} \mathrm{C}$ and at room temperature $25^{\circ} \mathrm{C} \pm 2^{\circ} \mathrm{C}$ for 1 month [1].

\section{RESULTS AND DISCUSSION}

\section{Suspending vehicle formula}

Microcrystalline cellulose, sodium carboxymethylcellulose, xanthan gum, and carrageenan were selected as suspending agents because the Ora-Plus vehicle contains these four suspending agents. For comparisons, formulations were prepared using two variations of suspending materials. Other ingredients that are used as additives in the formulation of suspending vehicles include calcium sulfate as a flocculating agent, methylparaben and potassium sorbate as preservatives, citric acid and sodium dihydrogen phosphate as buffers, dimethicone as an antifoaming agent, propylene glycol as stabilizing agent, and aquadest as a solvent.

\section{Evaluations of suspending vehicle \\ Sedimentation volumes}

Observations of sedimentation volumes in the present seven suspending vehicle formulations were performed after storage for $1,5,10,15,20$, 25 , and 30 days (Table 3 ).

The sedimentation volume of formula A indicated optimal vehicle characteristics, with no sedimentation at any of the observation times. These properties are in accordance with those of good suspensions, particularly because the particles in the vehicle were homogenously suspended and did not sediment quickly. Sedimentation rates are inversely proportional to vehicle viscosities. Thus, greater viscosity prevents the formations of sediments from suspension, as indicated by the lower sedimentation volumes seen in experiments with formula A.

The sedimentation volume in formula $\mathrm{C}$ was low at $14 \%$, although this value may in part reflect the density of the formed sediment. Likewise, formula B had a low sedimentation volume at $22 \%$, and similar to formula $\mathrm{C}$ formed compact and hard caked sediments after prolonged storage. Although these sedimentation volumes were small, they were unfavorably difficult to disperse, indicating physical instability of the suspensions.

Formula $\mathrm{E}$ had the second lowest sedimentation value after formula C, and after addition of DTZ tablets, weak interactions with suspending agents allowed rapid sedimentation. In contrast with the aforementioned formulas that form hard cake sediments, sediments from formula $\mathrm{E}$ tended to be stable for short times and were easier to resuspend with shaking. Hence, given the sedimentation volume after the addition of DTZ, the best formulation was formula E.

\section{Redispersion}

During storage, the suspending vehicle precipitates and the formed sediment must be redispersed by shaking to ensure appropriate doses at delivery. Thus, we compared redispersion of suspending vehicle formulas to rectify the sedimented particle so that with shaking, the particles in the vehicle can be evenly dispersed again [9].
In comparisons of the seven formulas, those for which formed sediments could be dispersed by shaking were favored because they did not form caked sediments after 30 days of storage. Formula A was the easiest formula to regenerate because it formed less sediment than the other formulas. Formula A also had favorable redispersion characteristics of the vehicle. In contrast, formula B required the most agitation to disperse the sediment evenly, and the sediment formed hard cakes after longer storage periods. Redispersion of formulas was compared in terms of numbers of shakes required (Table 4).

\section{Viscosity tests}

Viscosity tests were performed on the seven suspending vehicle formulas with and without DTZ tablets. In these experiments, formula A had the greatest viscosity, reflecting its four suspending agents. In formula C, high viscosity was observed due to the combination of cellulose microcrystalline and xanthan gum, and the latter is known to have a high viscosity degree at low concentrations. Compared with formula E, which also comprises a mixture of xanthan gum and sodium carboxymethylcellulose, the viscosity of formula $\mathrm{C}$ was higher. These observations can be explained by the presence of cellulose microcrystalline at $3 \%$ in formula $\mathrm{C}$, compared with carboxymethylcellulose sodium at only $0.5 \%$ in formula $\mathrm{E}$. Furthermore, the viscosity produced by sodium carboxymethylcellulose in solutions was higher than that of carrageenan, reflecting the ease of expansion of the former following dispersion in hot water. Therefore, dispersion of sodium carboxymethylcellulose in hot water may be causal of the high viscosity of formulas B and F. When compared with formula B, however, formula $\mathrm{F}$ had lower viscosity than B formula, which contained $3 \%$ microcrystalline cellulose, whereas formula $\mathrm{F}$ only contained carrageenan at $0.75 \%$. Formula D had higher viscosity than formula G, despite having equal carrageenan contents. This can be explained by the presence of higher cellulosic microcrystalline contents in formula D then gum xanthan concentrations in formula G. Thus, in decreasing order of viscosity, the formulas are arranged as follows: C, E, B, F, D, and G (Table 5).

\section{Particle sizes}

D values (4.3) were calculated to indicate average particle sizes of formulations. Of the seven suspending vehicle formulas, formula $\mathrm{E}$ had the largest particle size, with a D value (4.3) of $234 \mu \mathrm{m}$ reflecting the mixture of sodium carboxymethylcellulose and xanthan gum (Fig. 1). Both of these suspending agents have high degrees of pseudoplasticity and form clear colloidal solutions when dispersed in water. Furthermore, in the formula G, which was a mixture of xanthan gum and carrageenan, the resulting particle size was larger than that of formula $\mathrm{C}$, which comprises a mixture of xanthan gum and microcrystalline cellulose. Xanthan gum also has a high degree of pseudoplasticity, but in comparisons of formulas G and C, particle sizes were greater in $G$ because the carrageenan was dispersed in warm water to form hydrocolloids. In contrast, dispersion of cellulosic microcrystalline in water results in comparatively dilute solutions. Formula D comprised a mixture of cellulose microcrystalline and carrageenan and had the smallest particle size, with a D value (4.3) of $41.9 \mu \mathrm{m}$.

\section{pH tests}

In $\mathrm{pH}$ tests of the vehicle, $\mathrm{pH}$ values decreased after addition of DTZ tablets to all seven suspending vehicle formulas (Fig. 2). These

Table 3: Percentage sedimentation volumes of suspending vehicle formulas

\begin{tabular}{|c|c|c|c|c|c|c|c|}
\hline \multirow{2}{*}{$\begin{array}{l}\text { Day of } \\
\text { observation }\end{array}$} & \multicolumn{7}{|c|}{ Sedimentation volume (\%) } \\
\hline & Formula A & Formula B & Formula C & Formula D & Formula E & Formula F & Formula G \\
\hline 1 & 0 & 22 & 10 & 70 & 22 & 0 & 0 \\
\hline 5 & 0 & 22 & 14 & 66 & 22 & 0 & 96 \\
\hline 10 & 0 & 22 & 14 & 64 & 20 & 0 & 94 \\
\hline 15 & 0 & 22 & 14 & 64 & 20 & 96 & 92 \\
\hline 20 & 0 & 22 & 14 & 64 & 20 & 96 & 86 \\
\hline 30 & 0 & 22 & 14 & 64 & 20 & 92 & 76 \\
\hline
\end{tabular}


Table 4: Redispersion of suspending vehicle formulas

\begin{tabular}{|c|c|c|c|c|c|c|c|}
\hline \multirow{2}{*}{$\begin{array}{l}\text { Days of } \\
\text { observation }\end{array}$} & \multicolumn{7}{|c|}{ Redispersion (Times) } \\
\hline & Formula A & Formula B & Formula C & Formula D & Formula E & Formula F & Formula G \\
\hline 1 & 1 & 6 & 6 & 3 & 4 & 2 & 1 \\
\hline 10 & 1 & 16 & 12 & 4 & 7 & 2 & 2 \\
\hline 15 & 1 & 16 & 12 & 4 & 7 & 2 & 2 \\
\hline 20 & 1 & 16 & 12 & 4 & 7 & 2 & 2 \\
\hline 25 & 1 & 16 & 12 & 4 & 7 & 2 & 2 \\
\hline 30 & 1 & 16 & 12 & 4 & 7 & 2 & 2 \\
\hline
\end{tabular}

Table 5: Mean viscosities of formulas A-G

\begin{tabular}{lllllll}
\hline Viscosity & Formula & & & & \\
\cline { 2 - 6 } & A & B & C & D & E & F \\
\hline Mean value suspending vehicle (cPs) & 586.67 & 116.67 & 125 & 88 & 114.67 & 97.33 \\
Mean value DTZ suspension (cPs) & 130.67 & 101.67 & 123 & 96 & 114.67 & 97.33 \\
\hline
\end{tabular}

DTZ: Diltiazem hydrochloride

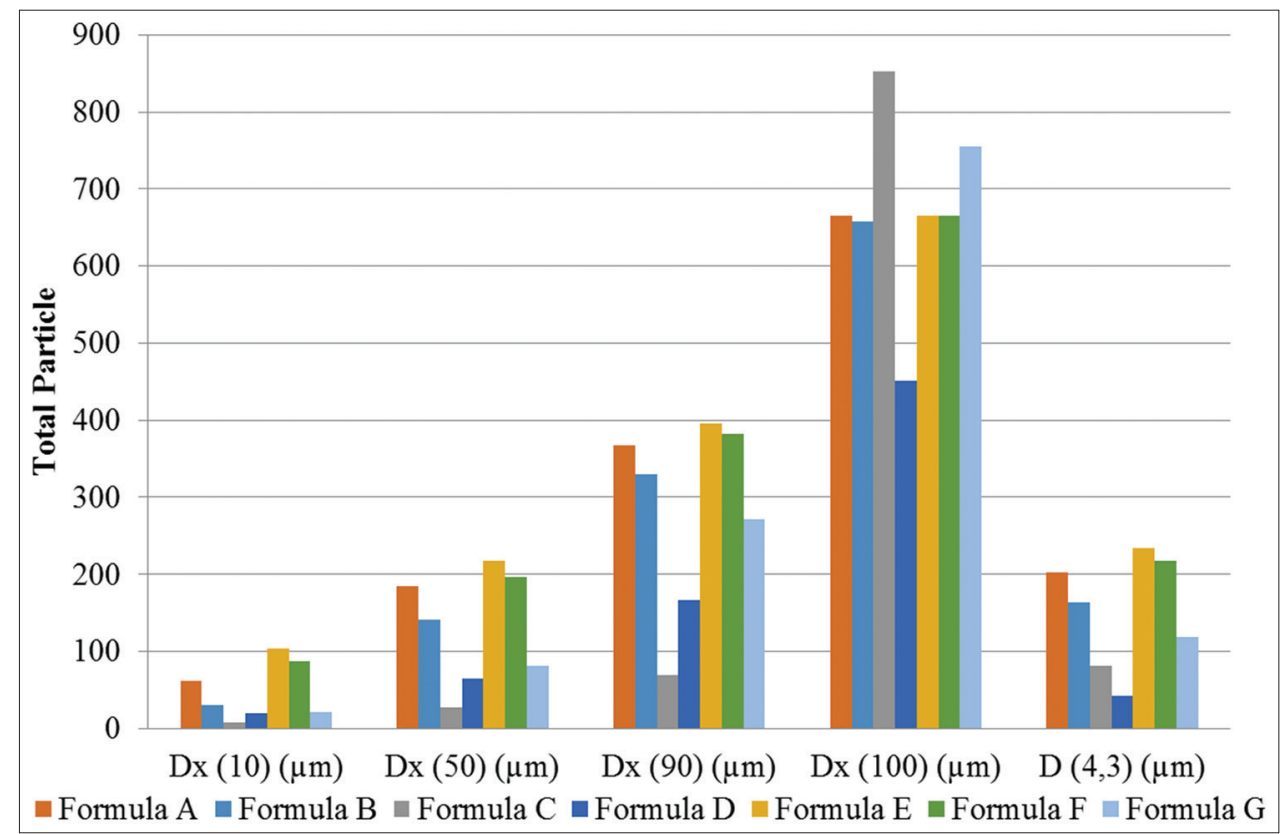

Fig. 1: Particle size distributions of formulas A-G

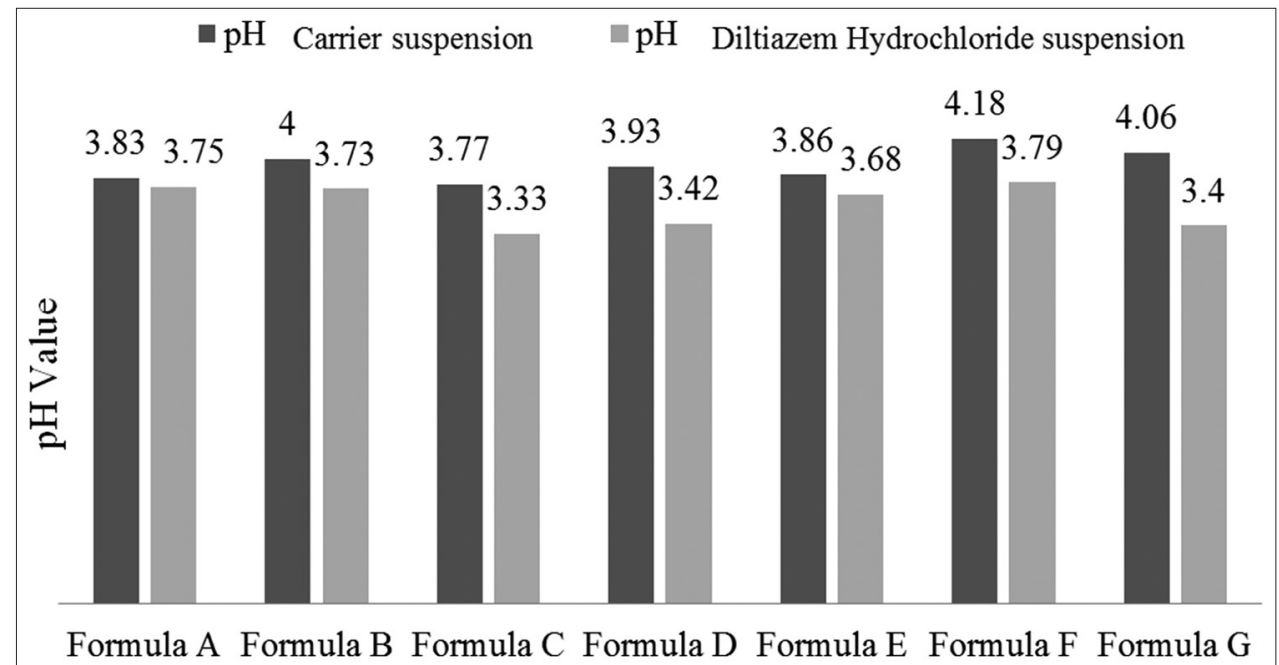

Fig. 2: Graph of pH ratios between suspending vehicle and suspension after addition of diltiazem hydrochloride tablets 
Table 6: Physical stability tests of Formulas A and E

\begin{tabular}{lll}
\hline \multirow{2}{*}{ Days } & $\mathbf{p H}$ & \\
\cline { 2 - 3 } & Formula A & Formula E \\
\hline 0 & 3.83 & 3.86 \\
3 & 3.78 & 3.68 \\
5 & 3.70 & 3.61 \\
10 & 3.62 & 3.60 \\
15 & 3.61 & 3.59 \\
20 & 3.58 & 3.57 \\
25 & 3.56 & 3.46 \\
30 & 3.50 & 3.47 \\
\hline
\end{tabular}

Table 7: Determinations of DTZ concentrations in suspensions

\begin{tabular}{llll}
\hline Condition & Formula & Days & $\begin{array}{l}\text { Concentration } \\
\text { (mean) (\%) }\end{array}$ \\
\hline Room temperature & Formula A & 0 & $75.11 \pm 8.426$ \\
& & 10 & $91.99 \pm 2.817$ \\
$4 \pm 2^{\circ} \mathrm{C}$ & Formula A & 30 & $84.11 \pm 0.964$ \\
& & 0 & $67.09 \pm 3.007$ \\
& & 10 & $90.67 \pm 8.614$ \\
& & 20 & $78.92 \pm 3.304$ \\
Room temperature & Formula E & 30 & $66.28 \pm 4.773$ \\
& & 10 & $83.54 \pm 1.315$ \\
& & 20 & $78.85 \pm 6.848$ \\
$4 \pm 2^{\circ} \mathrm{C}$ & Formula E & 30 & $62.21 \pm 5.066$ \\
& & 0 & $77.45 \pm 1.189$ \\
& & 10 & $83.61 \pm 1.769$ \\
& & 20 & $82.42 \pm 5.552$ \\
& & 30 & $65.97 \pm 2.726$ \\
\hline
\end{tabular}

DTZ: Diltiazem hydrochloride

decreases in pH are due to the acidity of DTZ tablets, which produced $\mathrm{pH}$ values of 4.3-5.3 in suspending vehicles [14].

\section{Physical stability tests}

Color and odor observations of formulas $\mathrm{A}$ and $\mathrm{E}$ showed no changes from day 0 to day 30 of storage (Table 6). Formula A was white and had a distinctive odor, and $\mathrm{E}$ formula was similarly white and had a similar drug odor. The absence of changes during storage suggests that formulas A and $\mathrm{E}$ remain stable at room temperature, even after the addition of DTZ tablets.

Decreasing $\mathrm{pH}$ values in formulas $\mathrm{A}$ and $\mathrm{E}$ during storage may be due to hydrolysis processes in suspensions. In particular, carrageenan is susceptible to hydrolysis and in water is hydrolyzed by acid, leading to further $\mathrm{pH}$ decreases.

Physical stability tests of the drug agent showed no changes during decreases in $\mathrm{pH}$ of formulas $\mathrm{A}$ and $\mathrm{E}$ at room temperature, indicating stability of the agent.

\section{Determination of drug concentrations}

\section{Determination of DTZ tablet concentrations}

DTZ tablet concentrations were determined using UV-visible spectrophotometry at the wavelength maxima of $236.0 \mathrm{~nm}$ and $10 \mathrm{ppm}$ [13]. From concentrations of $6.18,8.24,10.3,12.36,14.42$, and $16.48 \mathrm{ppm}$, the standard curve was described by the linear regression equation $\mathrm{y}=0.0444 \mathrm{x}+0.0132(\mathrm{r}=0.9989)$.

Using this standard curve for DTZ, DTZ contents of $30 \mathrm{mg}$ tablets were determined in triplicate, revealing $99.279 \%, 101.306 \%$, and $103.333 \%$ of the 30-mg added.

\section{Determination of DTZ concentrations in suspensions}

DTZ concentrations were determined in suspensions using UV-visible spectrophotometry. Samples of suspending vehicle containing 30-mg
DTZ tablets were analyzed after $0,10,20$, and 30 days of storage at room temperature $\left(25^{\circ} \mathrm{C} \pm 2^{\circ} \mathrm{C}\right)$ and in a refrigerator $\left(4^{\circ} \mathrm{C} \pm 2^{\circ} \mathrm{C}\right)$.

Absorption spectra of DTZ differed between the $1^{\text {st }}$ and past days of storage, particularly in formulations formula $\mathrm{A}$ and $\mathrm{E}$ at room temperature and in the refrigerator (Table 7). These data indicate that determinations of drug contents are not quantitative and suggest that the long extraction process using chloroform, which was performed with $24 \mathrm{~h}$ evaporation, introduces other compounds that can absorb at the same maximum wavelength, thus influencing determinations at the time of analysis. In addition, differences in the assay results may reflect a temporary diffusion process between the suspension and the organic chloroform phase during shaking for $1 \mathrm{~min}$. This may decrease concentrations of active substance, with active ingredient remaining in the chloroform fraction. Nonetheless, chloroform is required to separate active ingredient from suspensions for the determinations of concentrations of DTZ in suspensions.

\section{CONCLUSION}

The present physical stability test results show that our suspension carrier is physically stable for 30 days storage at room temperature, but that DTZ formulations were chemically unstable. In comparisons of the present formulations, the best suspending oral vehicle for DTZ was formula A.

\section{CONFLICTS OF INTEREST}

All authors declare that they have no conflicts of interest.

\section{REFERENCES}

1. Garg A, Garg S, She RW. Development of an extemporaneous oral liquid formulation of oxandrolone and its stability evaluation. Burns 2011;37:1150-3.

2. Patel VP, Desai TR, Chavda BG, Katira RM. Extemporaneous dosage form for oral liquids. Pharmacophore 2011;2:86-103.

3. Wiedyaningsih C, Hakimi M, Soenarto Y, Suryawat S. The use of the theory of planned behavior to predict factors influencing physician's decision to prescribe extemporaneous compounding dosage form for pediatric outpatients. Asian J Pharma Clin Res 2016;9:288-91.

4. Masareddy RS, Aloknath G, Kurien D. Development and characterization of diltiazem hydrochloride pulsatile drug delivery system for chronomodulated therapy. Asian J Pharma Clin Res 2011;4:168-73

5. Benzi JR, Mastroianni PD. Analysis of extemporaneous oral liquid from commercially available drugs in hospital. Braz J Pharm Sci 2016;52:517-25.

6. Muśko M, Sznitowska M. Stability of extemporaneous pediatric oral liquids compounded from tablets and drug substance: Case of propranolol and theophylline. Acta Pol Pharm 2013;70:137-45.

7. Khrisna A, Mohanan S. Formulation and evaluation of oral liquid suspension of paracetamol using newly isolated and characterized Hygrophila spinosa seed mucilage as suspending agent. Asian J Pharm Clin Res 2018;11:437-41.

8. Junyaprasert VB, Manwiwattanakul G. Release profile comparison and stability of diltiazem-resin microcapsules in sustained release suspensions. Int J Pharm 2008;352:81-91.

9. Nadaf SJ, Mali SS, Salunkhe SS, Kamble PM. Formulation and evaluation of ciprofloxacin suspension using natural suspending agent. Int J Pharm Sci Res 2014;5:63-70.

10. Senthil V, Sripreethi D. Formulation and evaluation of paracetamol suspension from Trigonella foenum graecum mucilage. J Adv Pharm Educ Res 2011;1:225-33.

11. Indonesian Ministry of Health. Indonesian Pharmacopeia. $4^{\text {th }}$ ed. Jakarta: Indonesian Ministry of Health; 1995.

12. Kuntawar RD, Mulgund SV. UV spectrophotometric estimation of diltiazem hydrochloride in bulk and tablet dosage form. World J Pharm Pharm Sci 2014;3:634-41.

13. Rahman N, Hejaz-Azmi SN. Spectrophotometric determination of diltiazem hydrochloride with sodium metavanadate. Microchem J 2000;65:39-43

14. Indonesian Ministry of Health. Indonesian Pharmacopeia. $5^{\text {th }}$ ed. Jakarta: Indonesian Ministry of Health; 2014. 